6-1-1989

\title{
An Improved Correction Algorithm for Number Density Measurements Made with the Forward-Scattering Spectrometer Probe
}

James A. Lock

Cleveland State University, j.lock@csuohio.edu

Edward A. Hovenac

Follow this and additional works at: https://engagedscholarship.csuohio.edu/sciphysics_facpub

Part of the Physics Commons

How does access to this work benefit you? Let us know!

\section{Publisher's Statement}

Copyright 1989 American Institute of Physics. This article may be downloaded for personal use only. Any other use requires prior permission of the author and the American Institute of Physics. The following article appeared in Review of Scientific Instruments 60 (1989): 1143-1153 and may be found at http://link.aip.org/link/doi/10.1063/1.1140329

\section{Original Citation}

Lock, James A. and Edward A. Hovenac. "An Improved Correction Algorithm for Number Density Measurements Made with the Forward-Scattering Spectrometer Probe." Review of Scientific Instruments 60 (1989): 1143-1153.

\section{Repository Citation}

Lock, James A. and Hovenac, Edward A., "An Improved Correction Algorithm for Number Density Measurements Made with the Forward-Scattering Spectrometer Probe" (1989). Physics Faculty Publications. 59.

https://engagedscholarship.csuohio.edu/sciphysics_facpub/59

This Article is brought to you for free and open access by the Physics Department at EngagedScholarship@CSU. It has been accepted for inclusion in Physics Faculty Publications by an authorized administrator of EngagedScholarship@CSU. For more information, please contact library.es@csuohio.edu. 


\title{
An improved correction algorithm for number density measurements made with the Forward Scattering Spectrometer Probe
}

\author{
James A. Lock \\ Fhysics Department, Cleveland State University, Cleveland, Ohio 44115 \\ Edward A. Hovenac \\ Sverdrup Technology Inc., NASA Lewis Research Center, Cleveland, Ohio 44135 \\ (Received 27 October 1988; accepted for publication 8 February 1989)

\begin{abstract}
A correction factor to the number density measured by the Forward Scattering Spectrometer Probe (FSSP) which compensates for dead time and coincidence errors was determined by calculating the probablities of and the average number of particles in the six possible types of dead time and coincidence events. These probabilities and averages were calculated by means of a probabilistic model based on Poisson statistics. A Monte Carlo computer simulation of the FSSP operation was also carried out and the number density correction factor was compared with the Monte Carlo data. For an actual number density of $2000 / \mathrm{cm}^{3}$, it was found that the measured number density was of the order of $300 / \mathrm{cm}^{3}$.
\end{abstract}

\section{INTRODUCTION}

The Forward Scattering Spectrometer Probe (FSSP), manufactured by Particle Measuring Systems, Inc, is standardly employed in measuring number densities and size distributions of atmospheric aerosols in the $2-100 \mu \mathrm{m}$ range, such as the water particles within clouds. The operation of the FSSP is described in many places ${ }^{1,2}$ and for aerosol number densities less than a few hundred per $\mathrm{cm}^{3}$, the measured values of the number density and size distribution are beleved to be reasonably accurate. However, within clouds and wind tunnels, the number density may approach values of $1000 / \mathrm{cm}^{3}$ or more, and as a result, coincidence and dead time errors in the measured number density become important. Dead time losses occur when particles enter and leave the optical scattering volume during the $2-6 \mu$ s electronics dead time which follows the recording of an earlier particle that has passed through the scattering volume. The dead time error causes the FSSP to underestimate the number density, but in the idealized situation it causes no distortion in the measured size distribution. (In actuality, particles whose sizes are beyond the instrument range and which enter the scattering volume saturate the amplifier. This causes baseline drift, lengthens the dead time, and thus distorts the measured size distribution.) Coincidence losses occur when a particle enters the scattering volume before an earlier particle has left. As a result, when several particles are in the scattering volume simultaneously, they are registered as a single larger particle. This causes the number density to be underestimated and causes the size distribution to be biased toward larger diameters.

Corrections to the measured number density due to dead time losses have been made by Baumgardner ${ }^{3}$ and $\mathrm{Cern}^{-4}$ and have been incorporated into the manufacturer's operation manual. ${ }^{5}$ This correction factor depends on the instrument's activity, i.e., the fraction of the total operation time spent in particles traversing the probe volume plus the subsequent dead times, and a constant $K$ which varies from probe to probe. This constant may either be measured experimentally ${ }^{4}$ or be predicted from the results of a computer program which simulates the FSSP operation. ${ }^{2,3}$ A more sophisticated correction incorporating both coincidence and dead time losses has been made by Baumgardner, Strapp, and Dye ${ }^{6}$ and by Julanov et al. ${ }^{7,8}$ This correction is based upon: (a) the assumption that the aerosol particles are distributed randomly in space and thus their arrival times at the scattering volume are described by Poisson statistics; and (2) that all the particles remain in the scattering volume for equal times. We call this the mean transit time nodel.

The number density correction proposed in this paper is an elaboration of the mean transit time model employed in Refs. 6-8. In addition to describing the arrival time by Poisson statistics, we also consider (1) the probability distribution describing the time duration that particles spend within the seattering volume and (2) an analysis of coincidence events in which some of the coincident particles are within the depth of fieid region of the scattering volume (DOF) and the remainder are outside the DOF. Feature (1) explicitly treats the probability distribution whose average value alone was employed in the mean transit time model. Feature (2) is important in that the FSSP measures the number density using only those particles which it records as passing through the DOF, otherwise known as the total number of strobes. In a coincidence event where some of the particles are within the DOF and the remainder are outside of it, a comparison of the amount of light scattered by each group determines whether the group passing through the DOF is recorded as a strobe or not. The importance of this class of coincidence events was noticed in Ref. 6 and we make a detailed analysis of it here.

Finally, it is of great importance to compare the activitybased number density correction of Refs. 3-5, the mean transit time model correction of Refs. $6-8$, and the present correction to actual experimental data or to some suitable approximation thereof. Since it is difficult to know beforehand the absolute number density within a cloud or wind 
tunnel, we have constructed a Monte-Carlo computer model of the FSSP operation and compared our corrections to simulated data produced by that model. The balance of this paper proceeds as follows. In Sec. I we describe the features of the FSSP scattering volume geometry and electronic circuitry which are used in the correction formulas. In Sec. II we explain our notation and derive the general form of the number density correction. In Sec.III we use a diagrammatic approach to calculate the various average values and probabilities that appear in the correction formula derived in Sec. II. We explain these calculations in detail both because the method of calculation is of great generality and because, with suitable modifications, it should be valid for the analysis of counting errors in the electronic circuitry of other instruments. In Sec. IV we propose an alternative determination of the actual number density which uses the instrument activity alone and which does not involve making any direct measurement of the number density. In Sec. $V$ we describe the Monte Carlo simulation of the FSSP operation, and finally in Sec. VI, we compare our correction formula to the resuits of the Monte Carlo calculation.

\section{FSSP OPERATION}

The optical scattering volume of the FSSP is the focal wais of a focused laser beam. It is taken to cylindrical with the diameter $d$. We assume that the light is of uniform intensity throughout the entire scattering volume. In actuality the scattering volume of an individual FSSP instrument may be somewhat different than this idealized geometry. We employ this geometry both because it is a zeroth-order approximation to the shape of the actual scattering volume and because resuits may actually be analytically calculated with this geometry using only a few input parameters. The final justification for its use depends on a comparison between the results of Sec. VI and actual FSSP data. We consider a rectangular coordinate system whose origin is at the center of the scattering volume and whose $Z$ axis coincides with the cylinder axis. The FSSP is mounted beneath the wing of an airplane and is flown so that the direction of the airspeed vector $v$ is along the scattering volume's $X$ axis. This is shown in Fig. 1. Forward scattered light from particles traversing

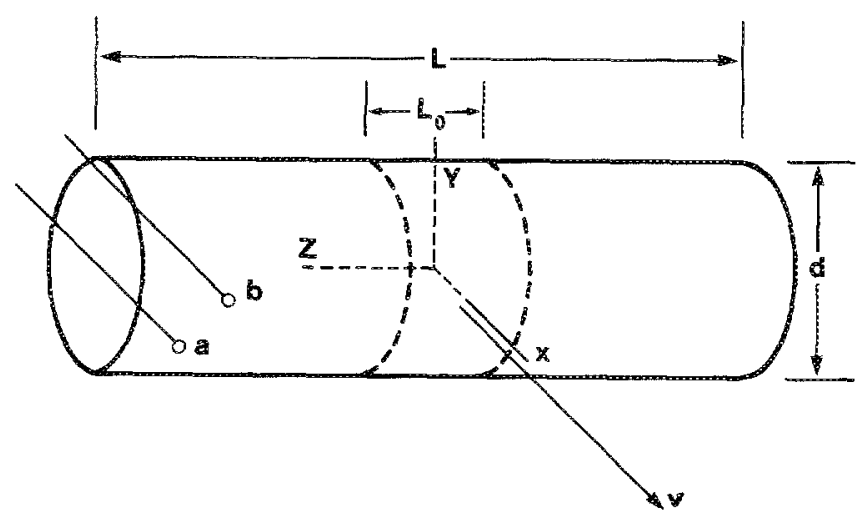

FIG. 1. The geometry of the FSSP optical scattering volume. The depth of feld is denoted by the dashed lines. Particle "a" passes through the widest part of the scattering volume and particle "b" passes through near the edge. the optical scattering volume is passed through a beam splitter and is incident on two photodiodes. Their output voltages are called ${ }^{1,2}$ the signal voltage $g_{s}(z)$ and annulus voltage $g_{a}(z)$. The purpose of the two voltages is to determine the extent of the DOF. This is indicated in Fig. 1 and we take its length to be $L_{0}$. Particles in the region beyond the DOF also scatter light into both the signal and annulus photodiodes, and trigger the instrument activity counter. The farther a particle is from the DOF, the less light it will send into the photodetectors. The end of the region of activity measurement is different for different size particles. We approximate the end of the scattering volume by the average of the locations of activity cutoff obtained for all the different size particles that are expected to be within the aerosol being measured. We take the total length of the scattering volume to be $L$. The length outside the DOF is then

$$
L_{12}=L-L_{0}
$$

We define $n_{s}$ to be the total number of strobes and $n_{f}$ to be the number of events occurring outside the DOF in the scattering volume. Then the total number of events registered is

$$
n_{e}=n_{s}+n_{f} \text {. }
$$

The total operation time of the instrument is $T$. The number density $\mathscr{N}_{s}$ (particles $/ \mathrm{cm}^{3}$ ) measured by the FSSP is given by

$$
\mathscr{N}_{s}=n_{s} / d L_{0} v T
$$

If $N$ is the total number of particles that enter the entire scattering volume in the time $T$, the actual number density $\mathscr{N}_{a}$ (particles $\left./ \mathrm{cm}^{3}\right)$ is given by

$$
\mathscr{N}_{a}=N / d L v T \text {. }
$$

Correspondingly, the average rate at which particles enter the scattering volume is

$$
\lambda=N / T \text {. }
$$

The major reason $n_{s}$ is incorrectly measured by the FSSP is that it takes the instrument a finite amount of time to analyze particles passing through the scattering volume. Whenever a strobe occurs, immediately after the particle or particles leave the scattering volume the electronics spend an amount of time $\tau_{s}$, the slow reset time, analyzing the event. During this time, additional particles entering the scattering volume are not detected. When a particle passes through the scattering volume outside the DOF, immediately after it leaves, the electronics spend an amount of time $\tau_{f}$, the fast reset time, analyzing the event. The purpose of the number density correction is to relate $\mathscr{N}_{s}$ to $\mathscr{N}_{a}$, or equivalently, to relate the total number of strobes to the total number of particles entering the entire scattering volume.

\section{THE NUMBER DENSITY CORRECTION: GENERAL CONSIDERATIONS}

The connection between. $\mathscr{T}_{s}$ and $\mathscr{T}_{a}$ is derived by considering the six different types of events that can occur in the FSSP scattering volume. These are shown in Figs. 2(a)2 (f). These figures show the signal photodiode voltage traveling down the electronics as a function of time toward the instrument's analysis circuitry. A voltage pulse begins when 
(a)

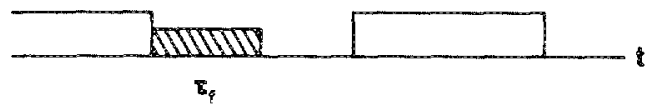

(b)

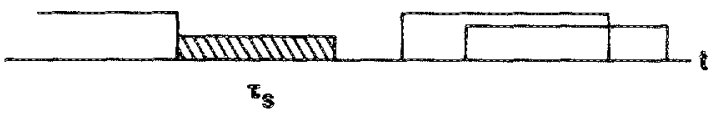

(c)

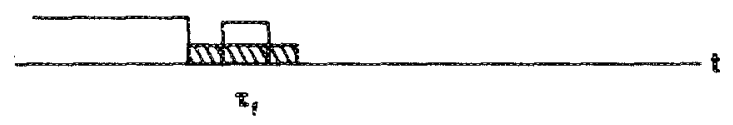

(d)

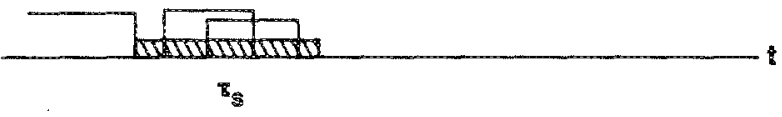

(e)

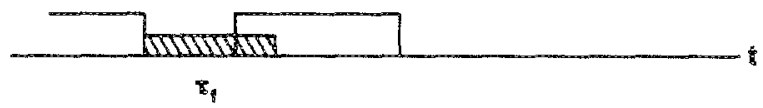

(i)

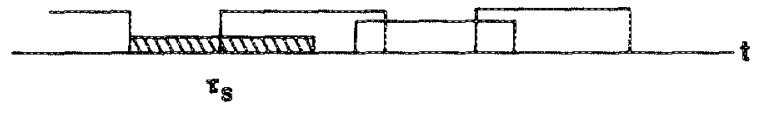

FIG. 2. (a), (b) Complete events following fast and slow resets; (c), (d) dead time events within fast and slow resets; and (e), (f) incomplete events starting in fast and slow resets.

a particle enters the scattering voiume and begins to scatter light. It end's when the particle leaves the scattering volume and ceases to scatter light. If a second particle enters the scattering volume before the first one leaves, they scatter light simultaneously, their two voltage pulses overiap, and they constitute a coincidence event. An event begins when the first particle in an $m$-particle coincidence cluster enters the scattering volume and the event ends when the last particle in the cluster leaves. The analysis circuitry records only the number of events. It cannot resolve a given event into the individual voltage pulses produced by its constituent particles.

The first two types of events we call complete events. These events correspond to particles which enter the probe volume after either a fast reset as in Fig. 2(a) or a slow reset as in Fig. 2(b) has been completed. A complete event may contain one or more particles. The average numer of part:cles per complete event is $\left\langle a_{c}\right\rangle$ and the total number of particles contained in ail the complete events recorded in the operation time is $N_{c}$.

The second two types of events we call dead time events. These events correspond to particles which completely pass through the scattering volume during a fast reset as in Fig. 2(c) or a slow reset as in Fig. 2(d). Again dead time events may contain one or more particles. The average number of dead time particles in a fast reset is $\left\langle a_{f d}\right\rangle$ and the average number in a slow reset $\left\langle a_{s d}\right\rangle$ where, in general,

$$
\left\langle a_{f d}\right\rangle \leqslant\left\langle a_{s d}\right\rangle
$$

since more particles can slip through undetected in a longer dead time than can in a shorter one. The total number of particles hidden in all the dead time events is $N_{d}$.

The last two types of everts are events that leak out the back of dead time intervals. We call these incomplete events. These events correspond to particles which enter the scattering volume during the dead time and are still within it when the dead time ends and the electronics reset. These events originate either in fast resets as in Fig. 2(e) or in slow resets as in Fig. $2(f)$. The average number of particles in an incomplete event that originates in a fast reset is $\left\langle a_{f i}\right\rangle$, the average number that originate in a slow reset is $\left\langle a_{s i}\right\rangle$, and the total number of particles contained in all the incompiete events is $N_{i}$. We have

$$
N=N_{i}+N_{a}+N_{i} \text {. }
$$

We may associate the following probabilities with these various types of events. We take $P_{f c}$ and $P_{f i}$ to be the probabilities that a fast reser is followed by a complete or an incomplete event, respectively. Similarly we take $P_{s c}$ and $P_{s i}$ to be the probabilities that a slow reset is followed by a compiete or an incomplete event, respectively. These probabilities satisfy

$$
P_{f c}+P_{f i}=1, \quad P_{s c}+P_{s i}=1 .
$$

We can estimate the fraction of recorded events that are strobes and events that oceur outside the DOF as

$$
\begin{aligned}
& n_{s}=\left(L_{0} / L\right) n_{e}-\left(L_{0} / L\right) n_{c} H, \\
& n_{f}=\left(L_{12} / L\right) n_{e}+\left(L_{0} / L\right) n_{e} H,
\end{aligned}
$$

respectively. The first term in these expressions contains the fraction of the total scattering volume inside and outside the DOF. If all the recorded events were one-particle events and the particles were randomly distributed along the $Z$ axis, these first terms alone would give the number of events occurring inside and outside the DOF. The factor $H$ in the second term of Eqs. (9) and (10) cescribes corrections to the one-particle event approximation due to the coincidence events in which one group of particles in the coincidence is within the DOF and the remainder are outside of it. In the limit of small number densities, $n_{c}$ is dominated by one-particle events and multiparticle coincidences are rare. Correspondingly, $H$ approaches zero in this limit.

All these quantities may now be combined to produce the connection between $\mathscr{N}_{s}$ and $\mathscr{T}_{a}$. The total number of particles in complete events may be written as

$$
\begin{aligned}
N_{c} & =\left\langle a_{c}\right\rangle\left(n_{s} P_{s c}+n_{f} P_{f c}\right) \\
& =\left\langle a_{c}\right\rangle n_{e}\left[\left(\frac{L_{0}}{L}-\frac{L_{0}}{L} H\right) P_{s c}+\left(\frac{L_{12}}{L}+\frac{L_{0}}{L} H\right) P_{f c}\right] .
\end{aligned}
$$

Similarly the total number of particles in dead time events and in incomplete events may be written as

$$
\begin{aligned}
N_{d} & =\left\langle a_{s d}\right\rangle n_{s}+\left\langle a_{f d}\right\rangle n_{f} \\
& =n_{e}\left[\left\langle a_{s d}\right\rangle\left(\frac{L_{0}}{L}-\frac{L_{0}}{L} H\right)+\left\langle a_{f d}\right\rangle\left(\frac{L_{12}}{L}+\frac{L_{0}}{L} H\right)\right]
\end{aligned}
$$


and

$$
\begin{aligned}
N_{i}= & \left\langle a_{s i}\right\rangle n_{s} P_{s i}+\left\langle a_{f i}\right\rangle n_{f} p_{f i} \\
= & n_{e}\left[\left\langle a_{s i}\right\rangle\left(\frac{L_{0}}{L}-\frac{L_{0}}{L} H\right) P_{s i}\right. \\
& \left.+\left\langle a_{f i}\right\rangle\left(\frac{L_{12}}{L}+\frac{L_{0}}{L} H\right) P_{f i}\right],
\end{aligned}
$$

respectively. Combining these with Eqs. (3), (4), and (7) we obtain

$$
\begin{aligned}
\mathscr{N}_{s}= & \mathscr{N}_{a}(1-H)\left[\left(\frac{L_{0}}{L}-\frac{L_{0}}{L} H\right)\right. \\
& \times\left(\left\langle a_{s d}\right\rangle+\left\langle a_{c}\right\rangle P_{s c}+\left\langle a_{s i}\right\rangle P_{s i}\right)+\left(\frac{L_{12}}{L}+\frac{L_{0}}{L} H\right) \\
& \left.\times\left(\left\langle a_{f d}\right\rangle+\left\langle a_{c}\right\rangle P_{f c}+\left\langle a_{f i}\right\rangle P_{f i}\right)\right]^{-\vdots}
\end{aligned}
$$

as the connection between the measured and actual number densities. The calculation of all the quantities appearing in this expression is carried out in Sec. III.

\section{THE NUMBER DENSITY CORRECTION: PROBABILISTIC CALCULATION}

Since we assume that all the aerosol particles that pass through the FSSP scattering volume are randomly distributed in space, the rate at which they enter the scattering volume parallel to the $X$ axis is given by Poisson statistics, i.e., the probability that $m$ particles enter the scattering volume during the time interval $t$ is given by

$$
R_{m}(t)=\left[(\lambda t)^{m} / m !\right] e^{-\lambda t} .
$$

In particular, the probability that none enter during the interval $t$ is $e^{-\lambda t}$ and the probability that one enters in the time interval $d t$ is $\lambda d t$. Since the incoming particles are randomly distributed in the $Y$ direction as well, those which pass through the center of the scattering volume such as particle " $a$ " in Fig. 1 remain in the beam for a longer time than those which pass through near the edge such as particle "b" in Fig. 1. If we assume that the scattering volume is the uniformly illuminated cylinder described in Sec. $\mathbb{I}$. and if

$$
\tau_{\max }=d / v
$$

is the maximum time that a particle can spend in the scattering volume, then one can show that

$$
Q(t) d t=\left\{\begin{array}{ccc}
\frac{t d t}{\tau_{\max }\left(\tau_{\max }^{2}-t^{2}\right)^{1 / 2}} & \text { for } & t \leqslant \tau_{\max } \\
0 & \text { for } & t>\tau_{\max }
\end{array}\right.
$$

is the probability that a particle remains within this idealized geometry scattering volume for an amount of time between $t$ and $t+d t$ after entering it. In this equation and throughout the balance of this article corrections to the transit time due to the finite size of the particles are not included. This probability distribution is normalized as

$$
\int_{0}^{\tau_{\max }} Q(t) d t=1
$$

and the average time a particle spends in the scattering volume is given by

$$
\tau_{\text {ave }}=\int_{0}^{\tau_{\max }} t Q(t) d t=\frac{\pi}{4} \tau_{\max }
$$

In order to evaluate the terms appearing in $\mathrm{Eq} .(14)$, we first consider the complete events. Let $P(m)$ be the probability that, given a complete event begins at $t=0$, it is an $m$ particle event. The voltage pulses corresponding to one-particle, two-particle, and three-particle complete events are given in Figs. 3(a)-3(i). Applying Eqs. (15) and (17) to every segment of the voltage pulses in Figs. $3(\mathrm{a})-3(\mathrm{c})$ and integrating over all the allowed voltage pulse durations and starting times we find that the probabilities of one-particle and two-particle events are given by

(a)

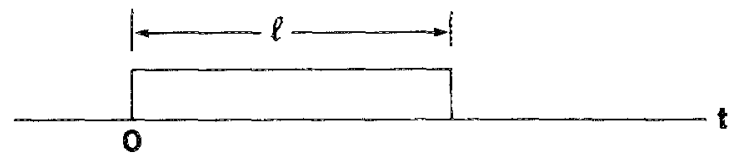

(b)

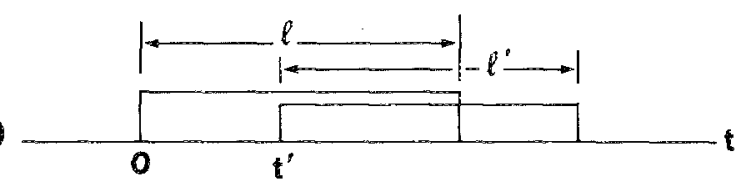

(c)
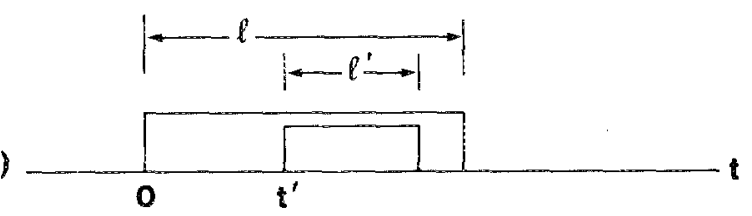

(d)

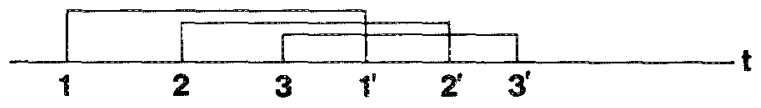

(e)

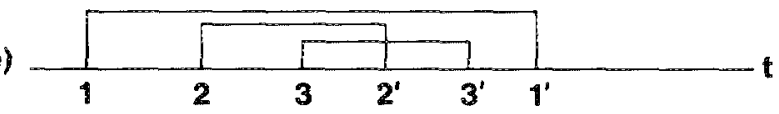

(n)

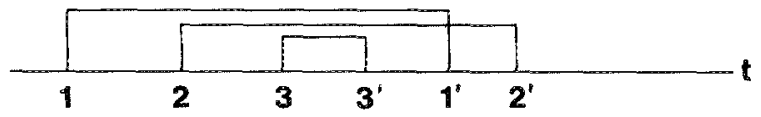

(g)

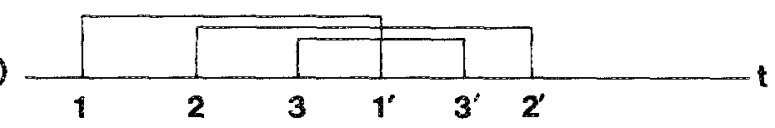

(h)

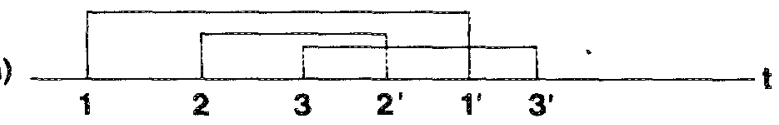

(i)

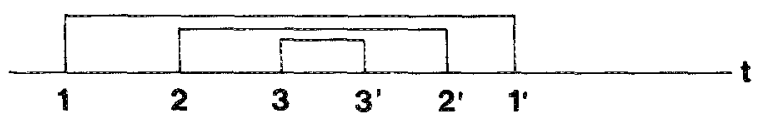

Fig. 3. (a) A one-particle complete event; (b), (c) the two types of twoparticle complete events; (d)-(i) the six types of three-particle complete events. The numerals 1,2 , and 3 denote the order in which the particles in (d)-(i) enter the scattering volume and the numerals $1^{\prime}, 2^{\prime}$, and $3^{\prime}$ denote the order in which they leave. 


$$
P(1)=\int_{l=0}^{\tau_{\operatorname{man}}} Q(l) d l e^{-\lambda l}
$$

and

$$
\begin{aligned}
P(2)= & \int_{l^{\prime}=0}^{\tau_{\max }} \int_{t^{\prime}=0}^{l} \int_{l^{\prime}=i-r^{\prime}}^{\tau_{\max }} Q(i) d l e^{-\lambda t^{\prime}} \\
& \times \lambda d t^{\prime} Q\left(l^{\prime}\right) d l^{\prime} e^{-\lambda l^{\prime}} \\
& +\int_{i=0}^{\tau_{\max }} \int_{t^{\prime}=0}^{l} \int_{l^{\prime}=0}^{i-t^{\prime}} Q(l) d l e^{-\lambda \lambda t^{\prime}} \\
& \times \lambda d t^{\prime} Q\left(l^{\prime}\right) d l^{\prime} e^{-\lambda\left(l-i^{\prime}\right)} .
\end{aligned}
$$

These equations may be understood as follows. Consider, for example, Fig. 3(b). The probability that a voltage pulse of duration $l$ begins at $t=0$ is $Q(l) d l$. The probability that no other pulse begins in the next time interval $t^{\prime}$ is $e^{-\lambda t^{\prime}}$. The probability that the second voltage pulse of duration $l$ ' begins within the time interval $d t^{\prime}$ centered about $t^{\prime}$ is $\lambda d t^{\prime} Q\left(l^{\prime}\right) d l^{\prime}$. The probability that no other pulse begins in the next time interval $l^{\prime}$ is $e^{-\lambda l}$. These factors give the integrand of the first term of Eq. (21). The duration of the first pulse may take on any value between 0 and $\tau_{\max }$. Assuming the second particle enters before the first particle leaves, its entering time $t^{\prime}$ can take on any value between 0 and $l$. Assuming further that the second particle leaves after the first one, then $l^{\prime}$ can be no shorter than $l-t^{\prime}$ and may be as long as $\tau_{\text {max }}$. These considerations give the limits of integration of the first term of Eq. (21). Using these rules to associate a probability with each segment of the appropriate voltage pulse diagram, the probability of any type of $m$-particle coincidence event of arbitrary complexity may be calculated in principle. This method of calculation is similar to the evaluation of probabilities and reaction rates in high energy physics by the use of Feynman diagrams. ${ }^{9,10}$

Using these diagrammatic rules, one can see that $P(3)$ consists of six, fivefold integrals as in Figs. 3(d)-3(i) and that things become quite complicated even for relatively low values of $m$. Assuming that all these integrals could be performed, one would find that

$$
\sum_{m=1}^{\infty} P(m)=1
$$

and that the average number of particles contained in a complete event is given by

$$
\left\langle a_{c}\right\rangle=\sum_{m=1}^{\infty} m P(m) .
$$

If one is lucky enough to have $P(m)$ being the elements of a geometric series, then

$$
P(m)=P(1)[1-P(1)]^{m \cdots 1}
$$

and

$$
\left\langle a_{c}\right\rangle=1 / P(1) .
$$

The biggest difficulty in using the diagrammatic approach is that none of the $P(m)$ integrals can be analytically evaluated using Eq. (17) for the probability distribution $Q(l) d l$. Even the simpiest of them, $P(1)$, can be evaluated only when the integrand is expanded in powers of $\lambda l$. Em. ploying the expansion parameter

$$
\alpha=\lambda \tau_{\max }=d^{2} L \mathscr{T}_{\alpha}
$$

and the results of Ref. 11 , we find that Eq. (20) with Eq. (17) inserted into it becomes

$$
\begin{aligned}
P(1)= & 1-\frac{\pi}{4} \alpha+\frac{2}{3} \frac{\alpha^{2}}{2 !}-\frac{3 \pi}{16} \frac{\alpha^{3}}{3 !}+\frac{8}{15} \frac{\alpha^{4}}{4 !}-\frac{5 \pi}{32} \frac{\alpha^{5}}{5 !} \\
& +\frac{16}{35} \frac{\alpha^{6}}{6 !}-\frac{35 \pi}{256} \frac{\alpha^{7}}{7 !}+\frac{128}{315} \frac{\alpha^{8}}{8 !}-\cdots
\end{aligned}
$$

As an alternative, one may approximate the $Y$-axis distribution of Eq. (17) as

$$
Q_{a}(t) d t=\left\{\begin{array}{ll}
\left(4 / \tau_{\text {nax }}^{4}\right) t^{3} d t & \text { for } t \leqslant \tau_{\max } \\
0 & \text { for } t>\tau_{\max }
\end{array}\right\}
$$

This approximate probability distribution has roughly the same shape as $Q(t) d t$ of Eq. (17), is properly normalized, and has an average value of 0.8 rather than $\pi / 4=0.7854$. Physically its use is justified by the fact that the actual FSSP scattering volume geometry might deviate somewhat from the idealized scattering volume geometry of Ea. (17). Using this form for $Q_{a}(1) d l$ in Eqs. (20) and (21), all the integrals can be performed analytically with the results

$$
\begin{aligned}
P(1)=4 I_{3} \approx & \frac{1}{4}-\frac{\alpha}{5}+\frac{\alpha^{2}}{12}-\frac{\alpha^{3}}{42}+\frac{\alpha^{4}}{192}-\frac{\alpha^{5}}{1080} \\
& +\frac{\alpha^{6}}{7200}-\frac{\alpha^{7}}{55440}+\frac{\alpha^{8}}{483840}-\cdots
\end{aligned}
$$

and

$$
\begin{aligned}
P(2)= & 4 I_{3}-16 I_{3}^{2}+4 I_{7}+\frac{16}{\alpha} I_{6}+\frac{48}{\alpha^{2}} I_{5} \\
& +\frac{96}{\alpha^{3}} I_{4}-\frac{24}{\alpha^{4}}+\frac{96}{\alpha^{4}} I_{3}+\frac{4}{5} \alpha I_{8} \\
\approx & \frac{4}{5} \alpha-\frac{74}{75} \alpha^{2}+\frac{246}{385} \alpha^{3}-\frac{727}{2520} \alpha^{4}+\cdots,
\end{aligned}
$$

where

$$
I_{k}=\frac{1}{\tau_{\max }^{k+1}} \int_{0}^{\tau_{\max }} l^{k^{k}} e^{-\lambda l} d l
$$

Last, in the mean transit time model, Baumgardner et $a l^{6}{ }^{6}$ and Julinov et $a l^{7,8}$ chose the $Y$-axis distribution as

$$
Q_{m t t}(t) d t=\delta\left(t-\tau_{\text {ave }}\right) d t .
$$

Using this distribution, the $P(m)$ may be calculated exactly for any $m \geqslant 1$ and they form the geometric series

$$
P(m)=e^{-B}\left(1-e^{\cdots B}\right)^{m-1},
$$

where

$$
\beta=(\pi / 4) \alpha \approx 0.7854 d^{2} L \mathbb{N}_{a} .
$$

This result is also given in Eq. (24) of Ref. 8.

It is of great interest to see whether the more realistic $Q_{a}(b) d l$ of $\mathrm{Eq}$. (28) also gives rise to a geometric series for the $P(m)$ because if it does not, we will be hard pressed to evaluate the infinite series of Eq. (23) given the fact that the calculation of the $P(m)$ for large values of $m$ becomes prohibitively laborious. As a test whether Eqs. (29) and (30) might behave as the first two terms of a geometric series, a comparison between $P(2)$ and $P(1)[1-P(1)]$ of Eqs. (29) and (30) shows the two quantities to be identical for 
small $\alpha$ and to be in agreement with each other to within $5 \%$ for $\alpha$ as large as $\alpha=1.69$ or $\beta=1.33$. Thus we believe that the geometric series approximation is reasonabiy accurate up to this value of $\alpha$, and that in this region $\left\langle a_{c}\right\rangle$ is given by Eq. (25) with $P(1)$ evaluated from Eq. (27).

This diagrammatic method of calculation may be applied to the other averages and probabilities appearing in $\mathrm{Eq}$. (14). For example, let $S(m)$ be the probability that $m$ particles pass completely through the scattering volume during a single dead time of duration $\tau$. Voltage pulse diagrams corresponding to $S(1)$ and $S(2)$ are given in Figs. 4(a)-4(e). The average number of particles that pass through undetected during the dead time is then

$$
\left\langle\alpha_{\tau d}\right\rangle=\sum_{m=0}^{\infty} m S(m) / \sum_{m=0}^{\infty} S(m)
$$

Using $Q_{a}(l) d l$ and $Q_{m t t}(l) d l$ of Eqs. (28) and (32), the terms $S(m)$ can be evaluated analytically for all values of $m$ with the results

(a)

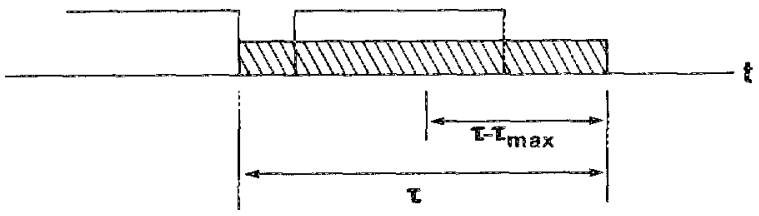

(b)

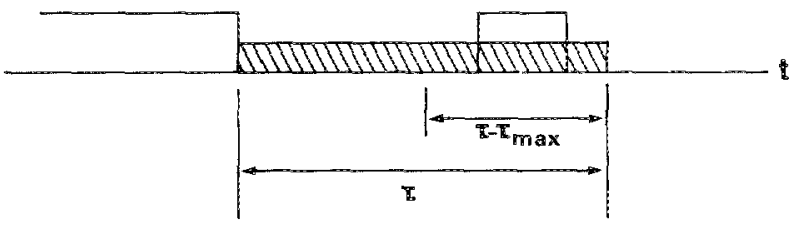

(c)

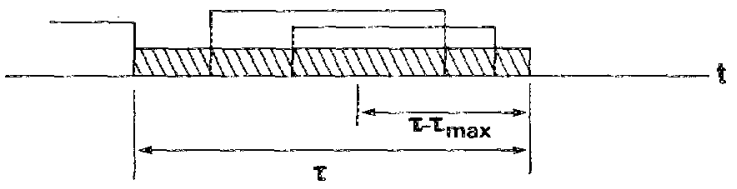

(d)

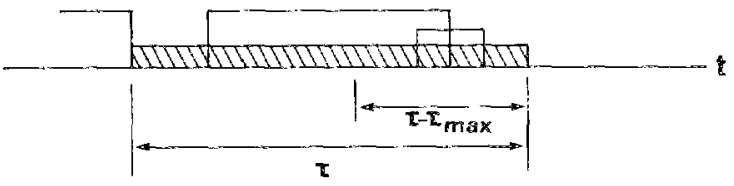

(e)

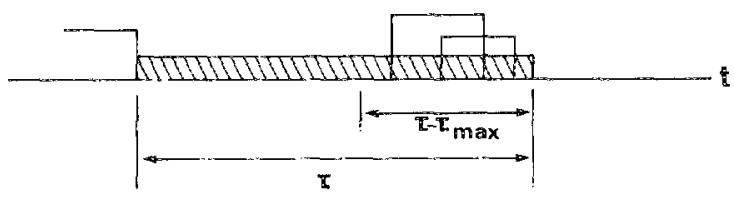

FIG. 4. (a), (b) The two types of one-particle dead time events. The probability $S(1)$ is the sum of these two diagrams; (c)-(c) the three types of twoparticle dead time events. The probability $S(2)$ is the sum of these three diagrams.

$$
S(m)= \begin{cases}\left(\lambda \tau-\frac{4}{5} \lambda \tau_{\max }\right)^{m} \frac{e^{-\lambda \tau}}{m !} & \text { for } \tau \geqslant \tau_{\max } \\ {\left[\frac{\lambda \tau}{5}\left(\frac{\tau}{\tau_{\max }}\right)^{4}\right]^{m} \frac{e^{\lambda \tau}}{m !}} & \text { for } \tau<\tau_{\max }\end{cases}
$$

and

$$
\left\langle a_{\tau d}\right\rangle=\left\{\begin{array}{ll}
\lambda\left(\tau-\frac{4}{5} \tau_{\max }\right) & \text { for } \tau \geqslant \tau_{\max } \\
(\lambda \tau / 5)\left(\tau / \tau_{\max }\right)^{4} & \text { for } \tau<\tau_{\max }
\end{array},\right.
$$

for $Q_{a}(l) d l$ of Eq. (28) and

$$
S(m)= \begin{cases}\left(\lambda \tau-\lambda \tau_{\text {ave }}\right)^{m}\left(e^{-\lambda \tau / m !)}\right. & \text { for } \tau \geqslant \tau_{\text {avc }} \\ 0 & \text { for } \tau<\tau_{\text {ave }}\end{cases}
$$

and

$$
\left\langle a_{\tau d}\right\rangle=\left\{\begin{array}{ll}
\lambda\left(\tau-\tau_{\mathrm{ave}}\right) & \text { for } \tau \geqslant \tau_{\mathrm{ave}} \\
0 & \text { for } \tau<\tau_{\mathrm{avc}}
\end{array},\right.
$$

for $Q_{m i}(l) d l$ of Eq. (32). The dead time interval $\tau$ is replaced either by the fast reset time $\tau_{f}$ or by the slow reset time $\tau_{s}$ in order to evaluate the dead time averages appearing in Eq. (14).

The calculation of the fraction of events that are complete and incomplete events proceeds in a similar way. Consider the last $\tau_{0}$ of a particular dead time interval. If the entire dead time interval $\tau$ is longer than $\tau_{\max }$ and $\tau_{0}=\tau_{\max }$, all particles entering the scattering volume before this cutoff time must leave before the dead time is over and cannot initiate an incomplete event. Particles entering the scattering volume during the last $\tau_{0}$ may or may not leave before the dead time is over. Thus they may or may not initiate an incomplete event. The probability that no particles enter during the last $\tau_{0}$ of dead time is equal to the probability that one will enter after the dead time is over and initiate a complete event. This is given by $e^{\lambda \tau_{0}}$. Thus the probability that one or more enter during the last $\tau_{0}$ of dead time is $1-e^{-\lambda \tau_{0}}$. But according to the form of $Q_{a}(t) d t$ of $\mathrm{Eq}$. (28), the probability that $m$ particles enter and leave during the last $\tau_{0}$ of dead time is $\left(\lambda \tau_{0} / 5\right)^{m}\left(e^{-\lambda \tau_{0}} / m\right.$ !). Summing this over $m$ gives $e^{-(4 / 5) \lambda \tau_{0}}-e^{-\lambda \tau_{5}}$ as the probability that one or more enter during the last $\tau_{0}$ and all leave before the electronics resets. Thus the probability of an incomplete event is $\left(1-e^{-\lambda \tau_{0}}\right)-\left(e^{-(4 / 5) \lambda r_{0}}-e^{\lambda \tau_{0}}\right)$. As a result, the fraction of all the events that are complete events and incomplete events is

$$
P_{\tau_{0} c}=e^{\lambda \lambda \tau_{0} /\left(1+e^{\lambda \tau_{11}}-e^{-(4 / 5) \lambda \tau_{0}}\right)}
$$

and

$$
P_{\tau_{0}, i}=\left(1-e^{-(4 / 5) \lambda \tau_{0}}\right) /\left(1+e^{-\lambda \tau_{0}}-e^{\left.(4 / 5) \lambda \tau_{i j}\right)},\right.
$$

respectively. The time interval $\tau_{0}$ is replaced by $\tau_{\max }$ for either the fast or slow resets if $\tau_{s}>\tau_{\operatorname{mix}}$ or $\tau_{f}>\tau_{\max }$ and it is replaced with the reset time itself and the $e^{-(4 / 5) \lambda \tau_{0}}$ factors are replaced by

$$
e^{-\lambda \tau_{0}} \exp \left[\frac{\lambda \tau_{0}}{5}\left(\frac{\tau_{0}}{\tau_{\max }}\right)^{4}\right]
$$

if $\tau_{s}<\tau_{\max }$ or $\tau_{f}<\tau_{\max }$, respectively. In the mean transit time model these probabilities are 


$$
P_{\tau_{10} c}=e^{-\lambda \tau_{0}}
$$

and

$$
P_{\tau, i}=1-e^{-\lambda \tau_{i n}}
$$

where again $\tau_{0}$ is replaced by $\tau_{\text {ave }}$ if the reset time is longer than $\tau_{a v e}$ and it is replaced by the reset time itself if the reset time is shorter than $\tau_{\text {ave }}$.

Compuring the average number of particles in an incomplete event is difficult because a simple voltage pulse diagram such as Fig. 5(a) represents a two-particle complete event but Fig. $5(b)$ does not represent a two-particle incomplete event. Rather Fig. 5(b) is interpreted as a one-particle incomplete event plus one dead time particle. As a result, the average number of particles per incomplete event should be slightly lower than the average number per complete event. However, diagrams such as Fig. 5(b) have a low probability of occurrence because the dead time particle in Fig. $5(\mathrm{~b})$ is unikely to remain within the scattering volume for times much shorter than $\tau_{\text {ave. }}$. This low probability is reffected in the slow increase of $Q(t) d t$ from 0 as $t$ increases from zero. As a result, if these low probability diagrams such as Fig. S(b) are double-counted, once as a two-particle incomplete event and once as a single dead time particle, then the average number of particles in complete and incomplete events will be identical and

$$
\left\langle a_{f i}\right\rangle=\left\langle a_{s i}\right\rangle=1 / P(1) .
$$

In the mean transit time model, the problematic diagrams such as Fig. $5(b)$ do not occur since all particles remain within the scattering volume for the same time $\tau_{\text {ave }}$ and thus they must leave in the order in which they arrived. In this model, these averages are

$$
\left\langle a_{f i}\right\rangle=\left\langle a_{s i}\right\rangle=e^{\beta}
$$

exactly as was found in Eq. (25) of Ref. 8 .

At this point, all the averages and probabilities may be combined in Eq. (14) to give

$\mathscr{N}_{s}=\frac{\mathscr{N}_{a} P(1)(1-H)}{1+\beta P(1)\left[\frac{L_{0}}{L} \frac{\tau_{s}}{\tau_{\text {ave }}}+\frac{L_{12}}{L} \frac{\tau_{f}}{\tau_{\text {ave }}}-1-H \frac{L_{0}}{L}\left(\frac{\tau_{s}-\tau_{f}}{\tau_{\text {ave }}}\right)\right]}$,

where we have assumed that $\tau_{s}>\tau_{\max }, \tau_{f}>\tau_{\max }$, and where we have replaced all factors of $4 \tau_{\max } / 5$ by $\tau_{\text {ave }}$ in order to try to compensate for our approximation of $Q(t) d t$ by $Q_{a}(t) d t$. In the mean transit time model, Eq. ( 14 ) becomes

$$
\mathscr{N}_{s}=\frac{\mathscr{N}_{a} e^{-\beta}(1-H)}{1+\beta e^{-\beta}\left[\frac{L_{0}}{L} \frac{\tau_{s}}{\tau_{\text {ave }}}+\frac{L_{12}}{L} \frac{\tau_{f}}{\tau_{\text {ave }}}-1-H \frac{L_{0}}{L}\left(\frac{\tau_{s}-\tau_{f}}{\tau_{\text {ave }}}\right)\right]} .
$$

The factor $H$ in Eqs. (9) and (10) describes the number of coincidence events that are judged to be within the DOF when some of the coincident particles are in the DOF and the remainder are not. Consider the one-particle, two-particle, and three-particle events of Figs. 6-8. For the one-particle events of Figs. $6(\mathrm{a})$ and $6(\mathrm{~b})$, if the particies are distributed randomly along the $Z$ axis and $n(1)$ is the total number of single-particle events, then $\left[L_{0} / L\right] n(1)$ of them are within the DOF as in Fig. 6(a) and $\left[L_{12} / L\right] n(1)$ of them are outside the DOF as in Fig. $6(\mathrm{~b})$. These correspond to the first term in Eqs. (9) and (10) discussed previously. For the twoparticle events of Figs. $7(\mathrm{a})-7(\mathrm{~d})$; if $n(2)$ is the total number of two-particle events, then $\left[L_{0} / L\right]^{2} n(2)$ of them as in Fig. $7(a)$ are registered as strobes, some fraction $f_{21}$ of the $2\left[L_{0} L_{12} / L^{2}\right] n(2)$ events of Figs. $7(\mathrm{~b})$ and $7(\mathrm{c})$ are regis-

(a)

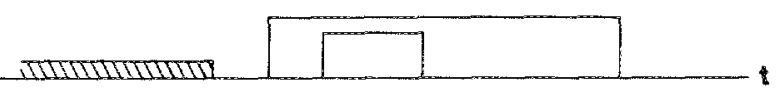

(8)

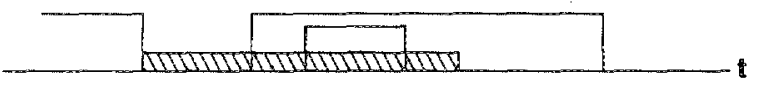

Fu. 5. (a) A two-particlc complete event; (b) a one-particle incomplete event accompanied by one dead time particle. tered as strobes, and none of the $\left[L_{12} / L\right]^{2} n(2)$ events of Fig. 7 (d) are registered as strobes. The three-particle events of Figs. $8(\mathrm{a})-8(\mathrm{~h})$ are handled similarly. If $n(3)$ is the total number of three-particle events, then $\left[L_{0} / L\right]^{3} n(3)$ of them as in Fig. $8\left(\right.$ a), some fraction $f_{32}$ of the $3\left[L_{0}{ }^{2} L_{12} / L^{3}\right] n(3)$ events of Figs. 8(b)-8(d), some fraction of $f_{31}$ of the $3\left[L_{0} L_{12}{ }^{2} / L^{3}\right] n(3)$ events of Figs. $8(\mathrm{e})-8(\mathrm{~g})$, and none of the $\left[L_{12} / L\right]^{3} n(3)$ events of Fig. $8(h)$ are registered as strobes. The total number of strobes is then

$$
\begin{aligned}
n_{s}= & \frac{L_{0}}{L} n(1)+\left(\frac{L_{0}^{2}}{L^{2}}+2 f_{21} \frac{L_{0} L_{12}}{L^{2}}\right) n(2) \\
& +\left(\frac{L_{0}^{3}}{L^{3}}+3 f_{32} \frac{L_{0}^{2} L_{12}}{L^{3}}+3 f_{31} \frac{L_{0} L_{12}^{2}}{L_{3}}\right) n(3)+\cdots
\end{aligned}
$$

(a)

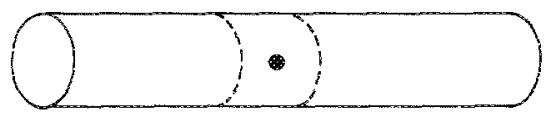

(b)

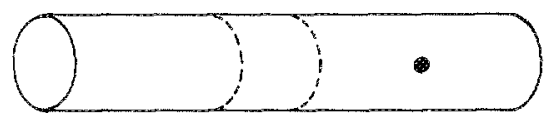

Fig. 6. One-particle events $(a)$ inside and (b) outside the DOF. 
(a)

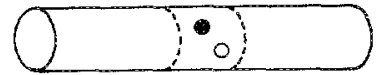

(b)

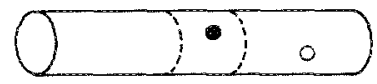

(c)

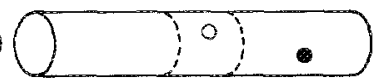

(d)

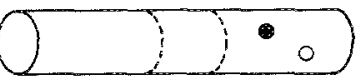

FIG. 7. Two-particle events with (a) both particles within the DOF, (b), (c) onc particle within the DOF and; (d) no particles within the DOF.

But the number of $m$-particle events is given by

$$
n(m)=n_{e} P(m) \text {. }
$$

If we employ Eq. (33) for $P(m)$ using the mean transit time model and Taylor series expand the result in powers of $\beta$ we obtain

$$
\begin{aligned}
n_{s}= & \frac{L_{0}}{L} n_{e}\left[1-\beta \frac{L_{12}}{L}\left(1-2 f_{21}\right)-\beta^{2} \frac{L_{12}}{L}\right. \\
& \times\left(\frac{L_{0}}{L}\left(2-3 f_{32}\right)+\frac{L_{12}}{L}\left(1-3 f_{31}\right)-\frac{3}{2}\left(1-2 f_{21}\right)\right) \\
& -\cdots]
\end{aligned}
$$

or

$$
\begin{aligned}
H= & \beta \frac{L_{12}}{L}\left[\left(1-2 f_{21}\right)+\beta\left(\frac{L_{0}}{L}\left(2-3 f_{32}\right)\right.\right. \\
& \left.\left.+\frac{L_{12}}{L}\left(1-3 f_{31}\right)-\frac{3}{2}\left(1-2 f_{21}\right)\right)+\cdots\right] .
\end{aligned}
$$

The determination of the fractions $f_{m n}$ of the various types of coincidence events that are registered as strobes depends on the signal voltage and annulus voitage factors $g_{s}(z)$ and $g_{a}(z)$ and on the sizes of the particles participating in the coincidence events. Thus a precise determination of the $f_{m n}$ is a very complicated undertaking. Baumgardener et al. ${ }^{6}$ assumed that $f_{m n}=n / m$, giving $H=0$ identically. This assumption is equivalent to saying either that one completely
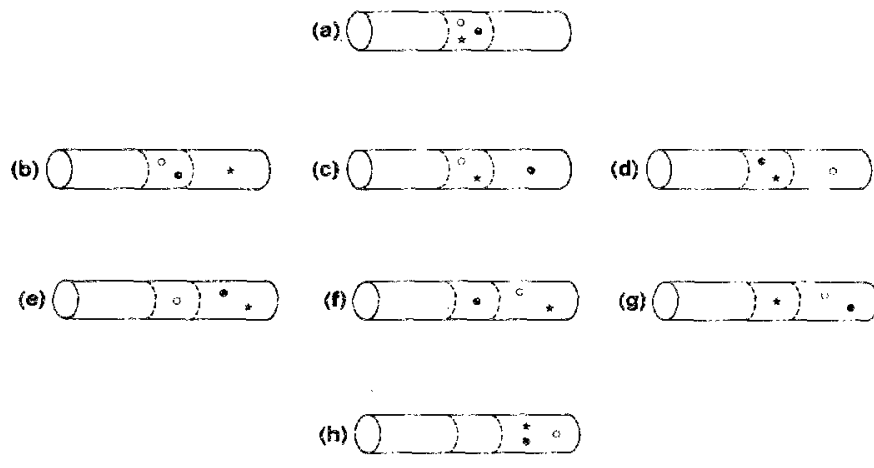

FIG. 8. Three-particle events with (a) three particles within the DOF; (b)(d) two particles within the DOF; (e)-(g) one particle within the DOF and; (h) no particles within the DOF. ignores the effect of $m$-particle coincidence events on determining the total number of strobes, or that whenever $m$ particles are coincident within the scattering volume, one is always larger than the rest and always dominates the situation. If the larger particle is within the DOF, the event is always counted as a strobe no matter where the other particles are. On the other hand, if the larger particle is outside the DOF, the event is never counted as a strobe no matter where the other particles are.

We choose to approximate $f_{m n}$ in a different way. For particles in the $5 \mu \mathrm{m}-50 \mu \mathrm{m}$ range, the near forward scattered light intensity is roughly proportional to the square of the particle radius. ${ }^{12}$ We consider an $m$-particle coincidence event where $n$ particles are within the DOF and $m-n$ particles are outside it. We choose to ignore the signal and annulus voltage factors and merely ask which cluster scatters more light. If the $n$ particles do, the event is registered as a strobe and if the $m-n$ do, the event is not registered as a strobe. Using this oversimplification to determine whether or not an event is recorded as a strobe, we find for a monodispersion that

$$
\begin{aligned}
& f_{21}=\frac{1}{2}, \\
& f_{31}=0, \quad f_{32}=1, \\
& f_{41}=0, \quad f_{42}=\frac{1}{2}, \quad f_{43}=1, \\
& f_{51}=f_{52}=0, \quad f_{53}=f_{54}=1, \quad \cdots,
\end{aligned}
$$

and that as a result

$$
\begin{aligned}
H_{m} & =\beta^{2} \frac{L_{12}}{L}\left(\frac{L_{12}-L_{0}}{L}\right)(1-\beta+\cdots) \\
& \approx \frac{L_{12}}{L}\left(\frac{L_{12}-L_{0}}{L}\right) \frac{\beta^{2}}{1+\beta} .
\end{aligned}
$$

Similarly, for a random distribution of sizes we find that

$$
\begin{aligned}
& f_{21}=\frac{1}{2}, \\
& f_{31}=\pi / 12, \quad f_{32}=1-f_{31}, \\
& f_{41}=\pi / 24, \quad f_{42}=\frac{1}{2}, \quad f_{43}=1-f_{41}, \cdots,
\end{aligned}
$$

and that as a result

$$
\begin{aligned}
H_{r} & =\beta^{2} \frac{L_{12}}{L}\left(\frac{L_{12}-L_{0}}{L}\right)\left[\left(1-\frac{\pi}{4}\right)-\beta\left(1-\frac{\pi}{3}\right)+\cdots\right] \\
& \approx 0.2146 \frac{L_{12}}{L}\left(\frac{L_{12}-L_{0}}{L}\right) \frac{\beta^{2}}{1+0.2146 \beta}
\end{aligned}
$$

Equations (53) and (55) require two comments. The first concerns our generalization from a two-term Taylor series expansion of $H$ to a rational function representation of $H$. Certainly for small $\beta$ this is justified and little error is introduced since the Taylor series expansion of Eq. (51) is rapidly convergent. For large $\beta$ the higher order terms of the Taylor series expansion become increasingly difficult to cal. culate and the convergence of the series becomes increasingly slow. Thus all we can do is hope that all of the complicated higher order terms add up to something simple and can be approximately described by the rational functions of Eqs. (53) and (55). Whether this hope is justified or not can only be determined by a comparison of $\mathrm{Eq} .(46)$ with experimental data. The second comment is that since $H$ is dependent on 
the width of the particle size distribution, the connection between $\mathscr{T}_{s}$ and $\mathscr{N}_{a}$ is also distribution dependent. This adds an additional ambiguity to the determination of the actual particle number density, since from the FSSP data, one knows only the measured size distribution width and not the actual size distribution width.

Equations (53) and (55) along with Eq. (46) is our connection between the measured and actual number density while Eq. (47) along with $H=0$ is the correction of Ref. 6. It should be mentioned that these are both complicated relations. Since $\beta$ and $\mathscr{N}_{a}$ are proportional to each other as seen in Eq. (34), $\mathscr{N}_{s}$ is not simply proportional to $\mathscr{N}_{a}$. It is rather a complicated function of $\mathscr{N}_{a}$, or equivalently of $\beta$, which will be seen in Sec. VI to be not uniquely invertible for all values of $\mathscr{N}_{s}$.

\section{THE ACTIVITY CORRECTION}

If the activity level of the FSSP is measured very accurately, one would not have had to go through the development of Secs. II and III since the activity can itself be written as a function of $\mathscr{T}_{a}$ or $\beta$. To do this we consider the mean transit time model of Eqs. (32) and (33). If we let $\left\langle\tau_{m}\right\rangle$ be the average duration of an $m$-particle event and

$$
\tau_{\text {reset }}=\frac{L_{0}}{L} \tau_{s}+\frac{L_{12}}{L} \tau_{f}
$$

is the average reset time after the compietion of an event, then the activity $A$ is given by

$$
A=\sum_{m=1}^{\infty} n_{e} P(m)\left(\left\langle\tau_{m}\right\rangle+\tau_{\text {reset }}\right) / \lambda / N
$$

Using Eqs. (7) and (11)-(13), this may be written as

$$
A=\frac{e^{-2 \beta} \sum_{m=1}^{\infty}\left(1-e^{-\beta}\right)^{m} \cdot 1\left(\lambda\left\langle\tau_{m}\right)+\lambda \tau_{\text {reset }}\right)}{1+\beta e^{-\beta}\left(\tau_{\text {reset }} / \tau_{\text {ive }}-1\right)} .
$$

The average duration of an $m$-particle event can be calculated in the following way. The total time spent in two-particle events may be written as $n_{e} P(2)\left\langle\tau_{2}\right\rangle$. But as seen by apply. ing the diagrammatic rules to Fig. $9(a)$, it may also be written as

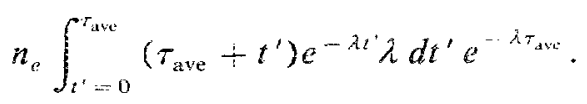

Similarly, the total time spent in three-particle events may be

(a)

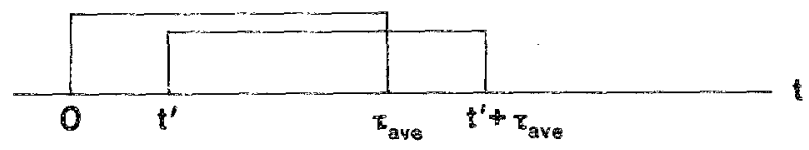

(b)

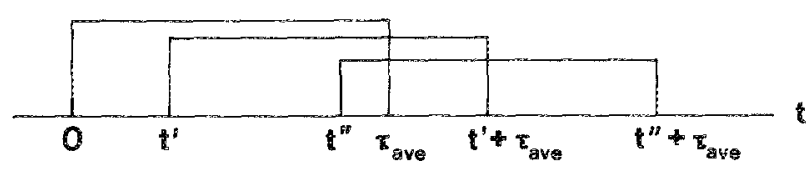

Fr. 9. (a) A two-particle complete or incomplete event and (b) a three particle complete or incomplete event in the mean transit time model. written by applying the diagrammatic rules to Fig. $9(\mathrm{~b})$ as

$$
\begin{aligned}
& n_{e} P(3)\left\langle\tau_{3}\right\rangle=n_{e} \int_{t^{\prime}=0}^{\tau_{\text {ave }}} \int_{t^{\prime \prime}=t^{\prime}}^{t^{\prime}+\tau_{\mathrm{uve}}}\left(\tau_{\mathrm{ave}}+t^{\prime \prime}\right) e^{\cdots \lambda t^{\prime}} \\
& \times \lambda d t^{\circ} e^{\lambda\left(t^{\prime \prime}-t^{\prime}\right)} \lambda d t^{\prime \prime} e^{-\lambda \tau_{\text {ive }}} \text {. }
\end{aligned}
$$

Generalizing this, performing the integrals, and expanding the result in powers of $\beta$, we obtain

$$
\begin{aligned}
\lambda\left\langle\tau_{m}\right\rangle= & \left(\frac{m+1}{2}\right) \beta-\left(\frac{m-1}{12}\right) \beta^{2} \\
& +\left(\frac{m-1}{720}\right) \beta^{4}+O\left(\beta^{6}\right)
\end{aligned}
$$

and

$$
\begin{aligned}
A= & {\left[\frac{\beta}{2}\left(1+e^{-\beta}\right)-\frac{\beta^{2}}{12}\left(1-\frac{\beta^{2}}{60}\right)\left(1-e^{-\beta}\right)\right.} \\
& \left.+\beta e^{-\beta}\left(\frac{\tau_{\text {reser }}}{\tau_{\text {ave }}}\right)\right] / \\
& {\left[1+\beta e^{-\beta}\left(\frac{\tau_{\text {reset }}}{\tau_{\text {ave }}}-1\right)\right] . }
\end{aligned}
$$

The first two terms of Eq. (61) were also derived in Eq. (31) of Ref. 7 . Again since $\beta$ is proportional to ${ }_{a}, \mathrm{Eq}$. (62) provides a connection between the activity and the actual concentration. However, in contrast to Eqs. (53) and (55), this function increases monotonically and poses no ambiguity for the inversion process.

The activity-based number density correction proposed by Baumgardner ${ }^{3}$ and Cerni ${ }^{4}$ employed the probe-dependent constant $K$ which could be either measured or computer-modeled. This constant may be exactly calculated from Eq. (62). For small $\beta$, Eq. (47) for the mean transit time model becomes

$$
\begin{aligned}
\mathscr{N}_{s} & =\frac{\mathscr{N}_{a}(1-\beta)}{1+\beta\left(\tau_{\text {reset }} / \tau_{\text {ave }}-1\right)} \\
& \approx \mathscr{N}_{a}\left[1-\beta\left(\tau_{\text {reset }} / \tau_{\text {ave }}\right)\right],
\end{aligned}
$$

and Eq. (62) for the activity becomes

$$
A=\beta\left(\tau_{\text {resel }} / \tau_{\text {ave }}+1\right) \text {. }
$$

Combining these equations and eliminating $\beta$ gives

$$
\mathscr{V}_{a}=\mathscr{H} /\left[1-A\left(\frac{\tau_{\text {reset }}}{\tau_{\text {resel }}+\tau_{\text {ave }}}\right)\right]
$$

or

$$
K=\tau_{\text {reser }} /\left(\tau_{\text {resct }}+\tau_{\text {ive }}\right) .
$$

The constant $K$ depends not only on the probe characteristics, but also on the airspeed through the factor $\tau_{\text {are }}$. For the University of Wyoming FSSP, Dye and Baumgardner ${ }^{2}$ have measured that $d=0.178 \mathrm{~mm}, \tau_{s}=5.6 \mu \mathrm{s}, \tau_{f}=2.1 \mu \mathrm{s}$, and $L_{0}=2.65 \mathrm{~mm}$. If $v=100 \mathrm{~m} / \mathrm{s}$ and $L \cong 12 \mathrm{~mm}$ as well, Eq. (66) gives $K=0.67$ which is roughly comparable to the values of $K=0.54$ measured by Cemi ${ }^{4}$ and $K=0.56$ obtained by a computer simulation by Dye and Baumgardner. ${ }^{2}$ 


\section{THE MONTE CARLO SIMULATION}

A Monte Carlo computer simulation was written in order to evaluate the performance of the FSSP density correction algorithm. The simulation was chosen over experimental testing for validation of the algorithm for two reasons. First, the simulation provided an unlimited number of test cases. This allowed rigorous testing of the algorithm using a variety of situations. Second, the experimental validation of the algorithm is difficult because the actual number density is not known to a high enough accuracy to effectively evaluate the algorithm.

The simulation program modeled every aspect of the operation of the FSSP except for the following simplifications. (1) All the droplets were assumed to be point sources of scattered light, (2) the laser beam profile in the simulation had only the gross features of the actual beam in the FSSP and thus the idealized scattering volume geometry was employed, and ( 3 ) the scattered light from the particles in the simulation was computed from Mie theory rather than using the "damped resonance" behavior seen in experimental testing. 'Each of these assumptions was studied and it was concluded that in most cases they had a minimal effect.

Modeled into the computer simulation (which was programmed on an IBM PC/AT) was virtually every aspect of the operation of the FSSP. This included the signal and annulus voltages as functions of particle position, reset times, beam diameter, transit time reject, instrument response to high velocity particles, coincidence events, and multiple particle scattering.

Input to the program were instrument parameters and environmental variables. The instrument parameters included the laser beam diameter, fast and slow reset times, instrument time-response factors, and a shape parameter for the laser beam profile. Environmental variables were the actual number density, the actual particle size distribution and the velocity of the particles. The program took these inputs and created a random three-dimensional distribution of simulated particles. These particles were allowed to pass through the simulated laser beam and the program analyzed how the FSSP would respond. The outputs of the computer simulation were the measured size distribution (in any of the four ranges of the FSSP), the total number of strobes, valid counts (which are used to calculate the particle size distribution), and the percent activity. The number density correction algorithm was then applied to the outputs to determine if it brought Monte Carlo data into closer agreement with the input values. This comparison is described in Sec. VI.

\section{COMPARISONS WITH THE MONTE CARLO CALCULATION}

The number density correction of Eq. (46) was calculated for

$$
\begin{aligned}
& d=0.023 \mathrm{~cm}, \\
& L_{0}=0.2 \mathrm{~cm}, \\
& L=1.6 \mathrm{~cm} \\
& \tau_{f}=2.3 \mu \mathrm{s},
\end{aligned}
$$

$$
\tau_{s}=6.0 \mu \mathrm{s},
$$

and

$$
v=100.0 \mathrm{~m} / \mathrm{s} \text {. }
$$

This value of the airspeed gives

$$
\tau_{\text {:ve }}=1.81 \mu \mathrm{s},
$$

and as a result, $\tau_{s}>\tau_{f}=\tau_{\max }$. This correction, as well as the mean transit time model correction of Eq. (47) and the activity-based correction of Eq. (65) with $K=0.6047$ from Eq. (66), are shown in Fig. 10. As is seen in Fig. 10, all models are identical for $\mathscr{N}_{a} \leqslant 500 / \mathrm{cm}^{3}$. Also, for $\mathscr{N}_{a} \gtrsim 500 / \mathrm{cm}^{3}$, there is a significant difference in the correction of Eq. (46) for a monodispersion $(m)$ and for a totally random distribution of sizes $(r)$. In Sec. III it was shown that for $\alpha \gtrsim 1.7$, the probabilities calculated in that section cease being accurately approximated by a geometric series. As a result, we cannot expect Eq. (46) to be valid past this cutoff value of $\alpha$. For the geometry of Eqs. (67)-(69) this corresponds to an actual number density of $2000 / \mathrm{cm}^{3}$.

The measured number density was obtained from the Monte Carlo simulation using samples of 250000 particles having a gaussian size distribution with an average diameter

$$
\bar{\alpha}=24.5 \mu \mathrm{m} \text {, }
$$

and with the root-mean-square width of the size distribution $\sigma$ being in the interval

$$
10^{-3} \leqslant \sigma / \bar{\alpha} \leqslant 0.71 \text {. }
$$

The lower end of this interval approximates a monodispersion and the upper end approximates a random distribution of sizes. The Monte Carlo data are also shown in Fig. 10. Neither the activity-based correction nor the mean transit

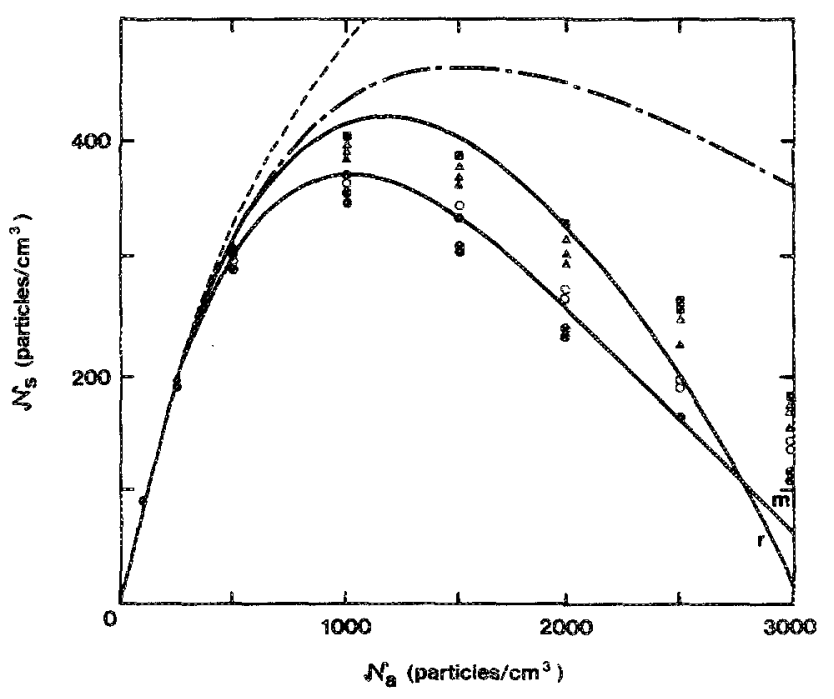

FIG. 10. The measured number density as a function of the actual number density for the FSSP parameters of Eqs. (67)-(72). The solid curves labeled $m$ and $r$ are the corrections of Eq. (46) for a monodispersion given by Fq. (53) and for a random size distribution given by Eq. (55), respectively. The dot-dashed curve is the mean transit time model correction of If. (47). The dotted curve is the activity-based correction of $\mathrm{Fa}$. (65) with $K==0.6047$. The data points are the results of the Monte Carlo calculation with the solid circles being $\sigma / \bar{\alpha}=10^{-3}$, the open circles being $\sigma / \bar{\alpha}=0.21$, the solid triangles being $\sigma / \bar{\alpha}=0.33$, the open triangles being $\sigma / \bar{\alpha}=0.46$, and the solid squares being $\sigma / \bar{\alpha}=0.71$. 


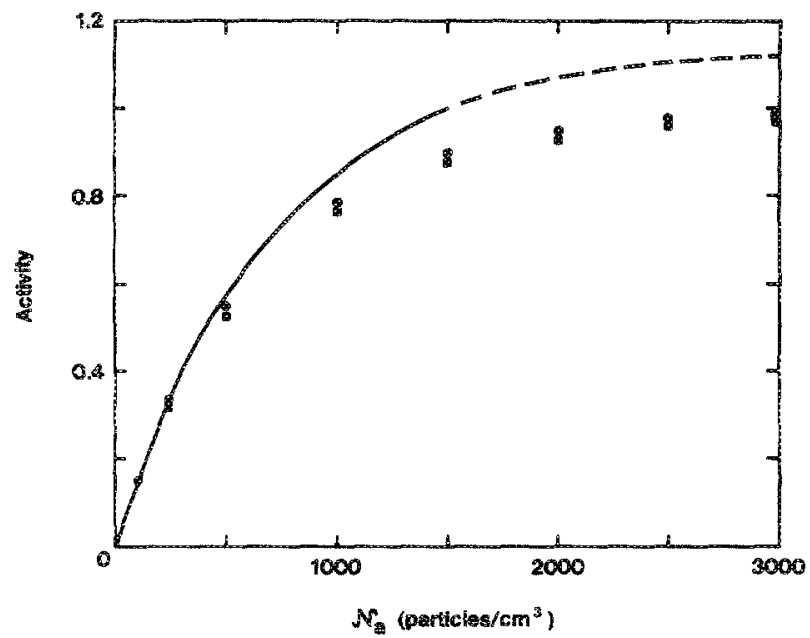

FIG. 11. The activity as a function of the actual number density from Eq. (62). The data points are the results of the Monte Carlo calculation with the solid circles being $\sigma / \vec{\alpha}=10^{-3}$ and the solid squares being $\sigma / \bar{\alpha}=0.71$.

time model correction fit the data for $\mathscr{N}_{a} \gtrsim 500 / \mathrm{cm}^{3}$. But the correction of Eq. (46) does fit this data until $\mathscr{N}_{a}>2000 / \mathrm{cm}^{3}$, the end of the theoretical region of validity of Eq. (46). Thus we claim that the correction of Eq. (46) represents a significant improvement over the corrections of the mean transit time and activity-based models. As was mentioned in Sec. I, the ultimate justification of the present probabilistic model rests on a comparison with actual FSSP data. Such a comparison is made dificult by the fact that actual number densities can only be inferred by a comparison with the measurements made by other types of instruments, each of which has its own biasings and errors. Such an experimental program is currently underway.

In Sec. IV, a relation between the activity and the actual number density was derived in the mean transit time model. For the parameters of Eqs. $(67)-(72)$ this is shown in Fig. 11 along with the Monte Carlo data for the size distribution of Eqs. (74) and (75). This figure requires three comments. First, the activity is a monotonically increasing function of $\mathscr{W}_{a}$ and is thus uniquely invertible. However, Eq. (62) becomes larger than unity for large $\mathscr{N}_{a}$ and fits the Monte Carlo data poorly for $\mathscr{N}_{a} \gtrsim 1000 / \mathrm{cm}^{3}$. Thus it should be of great interest to calculate the activity with the more realistic models of Eqs. (17) and (28) in order to attempt to improve the agreement with the Monte Carlo data. Second, in addition to the unique invertibility of Fig. 11, the activity is much more nearly independent of the width of the particle size distribution than is the measured number density of Fig. 10. This near independence of $\sigma$ is also an attractive feature when attempting to determine the actual number density from measured quantities. The last comment is that when obtaining $\mathscr{H}_{a}$ from the measured $\mathscr{H}_{s}$, both Figs. 10 and 11 should be used together. For most values of $\mathscr{N}_{s}$, there are two possible values of $\mathscr{A}_{a}$, one corresponding to a low number density and the other corresponding to a high one. The correct value of $\mathscr{T}_{a}$ is suggested from examining the activity, a low value of $A$ giving the lower value of $\mathscr{T}_{a}$ and a high value of $A$ giving the higher value of $\mathscr{H}_{a}$. Aiternatively, the value of $\mathscr{N}_{a}$ determined from the activity in Fig. 11 could be used as a starting value in an interative algorithm to determine the correct value of $\mathscr{N}_{a}$ in Fig. 10.

\section{ACKNOWLEDGMENTS}

One of us (J.A.L.) wishes to thank the Optical Measurement Systems Branch of the NASA Lewis Research Center for its hospitality and financial support during summer 1988 when this work was carried out under a NASA/ ASEE Summer Faculty Fellowship. The authors also wish to thank the referee for making the Julanov et al. papers known to them and for a number of insights on FSSP operation.

'R. G. Knollenberg, in International Conference on Cloud Physics (American Meieorological Society, Boulder, CO, 1976), pp. 554-561.

${ }^{2} J$. E. Dyc and D. Baumgardner, J. Atmos. Ocean. Technol. 1, 329 (1984). 'D. Baumgardner, J. Clim. Appl. Meteor. 22, 891 (1983).

${ }^{4}$ T. A. Cerni, J. Clim. Appl. Meteor. 22, 1346 (1983).

${ }^{5}$ Particle Measuring Systems, Inc., "Forward scartering spectrometer probe, PMS model FSSP-100, operating and servicing manual," May 1984, Appendix V.

${ }^{6} D$. Baumgardner, W. Strapp, and J. E. Dye, J. Almos. Ocean. Technol. 2, $626(1985)$

${ }^{7}$ Yu. V. Julanov, A. A. Lushnikov, and I. A. Nevskii, J. Aerosol Sci. 15, 69 (1984).

"Yu. V.Julanov, A. A. Iushnikov, and I. A. Nevskii, J. Aerosol Sci. 17, 87 (1986).

${ }^{\prime R}$. P. Feynman, $Q E D$, the Strange Theory of Light and Matter (Princeton University, Princeton, NJ, 1985), Chap. 3.

"J. J. Sakurai, Advanced Quantum Mechanics (Addison-Wesley, Reading, MA, 1980), Secs. 4.4-4.5.

${ }^{11}$ I. \$. Gradshteyn and I. M. Ryzhik, Table of Integrals, Series, and Products (Academic, New York, 1965), 2.271-2.274.

${ }^{12}$ H. C. yan de Hulst, Light Scattering by Small Particles (Dover, New York, 1981), Sees. 9.31, 9.4, and 12.32. 